\title{
Homebased Standing Core Exercise Training Improves Femoral Blood Flow but not Arterial Stiffness in Middle-Aged to Older Adults
}

\author{
Hsin-Fu Lin ${ }^{1, *}, \mathbb{D}$, Soun-Cheng Wang ${ }^{2}$, Hao-Min Cheng ${ }^{3,4, \mathbb{D}}$, Jun Sugawara ${ }^{5}$ \\ ${ }^{1}$ Department of Athletics, National Taiwan University, No 1, Sec 4, Roosevelt Rd., Taipei 10617, Taiwan \\ ${ }^{2}$ Department of Athletic Sport, National Chung Cheng University, Chiayi, Taiwan \\ ${ }^{3}$ Department of Medicine, National Yang-Ming University School of Medicine, Taipei, Taiwan \\ ${ }^{4}$ Center for Evidence-based Medicine, Taipei Veterans General Hospital, Taipei, Taiwan \\ ${ }^{5}$ Human Informatics Research Institute, National Institute of Advanced Industrial Science and Technology, Tsukuba, Japan
}

\section{ARTICLE INFO}

\section{Article History}

Received 13 October 2020

Accepted 15 December 2020

Keywords

Aerobic exercise

physical activity

resistance training

trunk

\begin{abstract}
Homebased exercise is often advocated to improve physical fitness in the aging population, especially when outdoor activity is not available. Yet we often have a limited understanding of its training effects for clinical uses.

Aim: To investigate a short-term exercise training of standing core exercise on femoral blood flow and vascular hemodynamic changes in middle-aged to the older population.

Methods: A total of 34 middle-aged to older adults were recruited and randomly assigned into walking $(\mathrm{M} / \mathrm{F}=6 / 7$, age $=$ $66 \pm 3$ years $)$, core exercise $(\mathrm{M} / \mathrm{F}=6 / 6$, age $=66 \pm 2$ years $)$, and the control group $(\mathrm{M} / \mathrm{F}=4 / 5$, age $=70 \pm 3$ years $)$. Carotid and brachial blood pressure, Carotid-femoral Pulse Wave Velocity (PWV) and Brachial-ankle PWV (baPWV), femoral blood flow and compliance, leg hemodynamics, as well as aerobic and muscular fitness (30 s arm curl and sit-to-stand test) were measured pre and post 8 weeks of exercise training (60-70\% HRmax, three sessions/week).

Results: Core exercise group significantly increased femoral blood flow, but not overall vascular functions; arm muscle endurance and leg power performance were also significantly improved after training. The walking group significantly reduced baPWV, leg resistance, as well as increase femoral blood flow and compliance after training. Compared with the control, the core group elicited a higher femoral blood blow, whereas leg resistance was significantly reduced in the walking group after training.

Conclusion: A short-term standing core training appears to augment femoral blood flow and muscular fitness in middle-aged to older adults, yet walking is more effective in improving overall vascular health in this population.
\end{abstract}

\section{HIGHLIGHTS}

- Standing core training improves femoral blood flow in middle-aged to older adults.

- The increase of blood velocity appears to associate with the improvement of blood flow.

- Walking exercise training is superior to improve overall vascular function.

(C) 2021 The Authors. Publishing services by Atlantis Press International B.V. This is an open access article distributed under the CC BY-NC 4.0 license (http://creativecommons.org/licenses/by-nc/4.0/)

\section{INTRODUCTION}

The reduction in peripheral blood flow has been shown associated with a loss of muscle mass with advancing age [1]. Lower shear stress resulted from attenuated blood flow along with the vessel wall is one of the critical underlying mechanisms in reducing nitric oxide availability and exacerbating vascular dysfunction and leg conductance [2], leading to arterial stiffening, high blood pressure and future cardiovascular events.

"Corresponding author. Email: hsinfu@ntu.edu.tw

Peer review under responsibility of the Association for Research into Arterial Structure and Physiology

Data availability statement: The data that support the findings of this study are available from the corresponding author $[\mathrm{HFL}]$, upon reasonable request.
Endurance exercise was demonstrated effectively in restoring blood flow with advancing age [3] via the increase in shear stress and endothelial function [4]. Resistance exercise is also a modality that acutely [5] and chronically [6] increase limb blood flow in addition to muscle strength and power improvement. Both types of exercise have been shown to lower blood pressure in the literature [7]. Unlike conventional exercise mainly involved limb movements, core exercise is often explicitly adopted to improve functional, stability, and mobility [8], as well as to maintain musculoskeletal health. Populations such as older adults [9] and individuals with lower back pain [10] could benefit from participating in core exercise. Importantly, the smooth neutral movement and relatively lower intensity characterize core exercise, which might be an appealing alternative for individuals of lower functional capacity. 
However, the extent to which core-biased exercise training induces changes in blood flow and vascular health remains unknown.

In the present study, we adopted a homebased standing core rotational exercise device that provides low-resistance from the platform against arm-assisted trunk rotation of participants while exercising (shown in Figure 1). This exercise performing in the standing position against spring-generated resistance mimics specific core muscle function pattern needed during daily tasks and sports activities [8]; the closed-chain movement may be preferable to people with knee complications [11], indicating its potential clinical use for older adults. Our unpublished data showed core exercise at optimal rotation rhythm for $30 \mathrm{~min}$ acutely increased relative blood flow and leg conductance in the young population. Accordingly, we aimed to test the hypothesis that standing core resistance endurance exercise could lower blood pressure, improve leg hemodynamics and muscular fitness after a short-term intervention by including walking and sedentary control groups in our study design.

\section{MATERIALS AND METHODS}

\subsection{Experimental Design}

After screening and pre-exercise measurement, participants were randomly assigned to walking, core training, or control group based on block randomization followed by an 8 -week intervention consisting of three supervised sessions (30 min each) per week for exercise groups. In contrast, the control group was asked not to engage in any form of exercise training. In every training session, the heart rate monitor was equipped to record and ensure the exercise intensity was achieved and maintained. To control for the effect of exercise and diet on dependent variables, physical activity log and 3-day dietary records were obtained before and after the intervention.

As shown in Figure 1, a standing core exercise machine equipped with adjustable resistance was used for this study (Core X. Fitcrew Inc., Taiwan). Participants were instructed to rotate their trunk within a set range of rotation (at least $140^{\circ}$ ). Handgrip assistance in the same position was allowed to guard against spring resistance $(13.5 \mathrm{~kg})$ produced by the machine. Hence, during exercise, participants were required to work against this spring resistance to achieve the required range of motion for $30 \mathrm{~min}$. Metronome rhythm was set at a cadence of 75-80 rpm, which was demonstrated to elicit $60-70 \%$ of age-predicted maximal heart rate (HRmax) in older adults by our pilot work. All participants gave their written informed consent, and all procedures were reviewed and approved by the Institutional Review Board of University Hospital.

\subsection{Participants}

Thirty-seven healthy middle-aged to older adults were recruited to complete a short-term exercise intervention. Only postmenopausal women were included as female participants to avoid the confounding effects of estrogen. Exclusions criteria included: (1) obesity (BMI $\left.>30 \mathrm{~kg} / \mathrm{m}^{2}\right)$, (2) cigarette smoking within past 6 months, (3) history of diabetes (fasting blood glucose $>126 \mathrm{mg} / \mathrm{dL}$ ) and heart disease, (4) injury that may prevent him or her from completing the exercise; and (5) use of over-the-counter supplements or vitamins. None of the subjects exercised (resistance or endurance training) more than three times a week, as determined by a physical
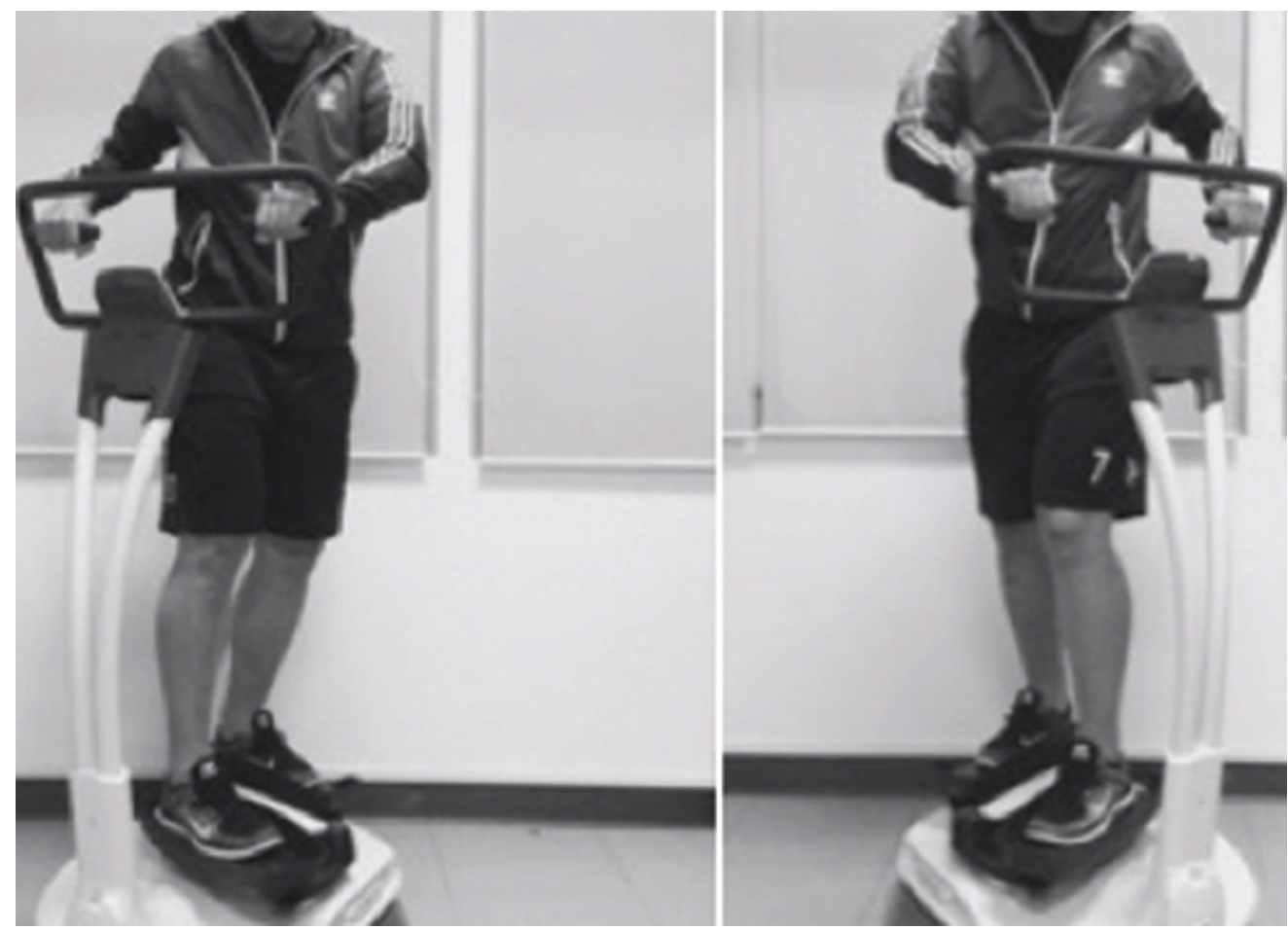

Figure 1 Scheme of stand core exercise (the subject has given permission for the use of this figure). 
activity questionnaire. Recruited participants had no history of hypertension $(<140 / 90 \mathrm{mmHg}$ ) or kidney dysfunction.

\subsection{Procedures}

A familiarization session was implemented to ease anxiety before the first testing session. Testing was conducted pre- and postexercise training intervention. All post-training measurements were performed 24-48 $\mathrm{h}$ after the last exercise session to avoid the immediate effects of a single bout of exercise. Participants were required three visits to the laboratory for pre- and post-training testing measurements performed approximately at the same time in the morning for each subject. Participants were instructed to arrive at the laboratory after a 12-h overnight fast in the last visit for the blood draw.

Both exercise groups underwent 8 weeks of intervention (30 min/ session, three sessions/week) at the intensity of 60-70\% HRmax. The cadence of core exercise and treadmill speed was adjusted accordingly. A heart rate monitor was worn to assess heart rate throughout the exercise training. Exercise sessions were monitored and supervised by an investigator to ensure the target heart rate had been reached, and all participants were able to complete the exercise session throughout the intervention.

\subsection{Measurements}

\subsubsection{Maximal oxygen uptake}

All participants underwent a treadmill test to determine individual maximal oxygen consumption $\left(\mathrm{VO}_{2} \mathrm{max}\right)$ by using the Balke protocol. Briefly, the male and female subjects started walking at 3.5 and $3.0 \mathrm{mile} / \mathrm{h}$ respectively and then increased the exercise intensity by increasing the slope by $3 \%$ every $2 \mathrm{~min}$ [12]. Participants were verbally encouraged to complete the test. Heart rate and subjective feelings of the exercise on the Borg's scale was recorded at every stage. Exercise termination criteria including volitional exhaustion, Borg's scale $>17$, or heart rate $>90 \%$ HRmax were used for testing safety concerns. The speed and slope of the subject's last minute were converted into metabolic equivalents using the American College of Sports Medicine [13].

\subsubsection{Blood pressure and arterial stiffness}

Participants were instructed to rest quietly in the supine position at least 10 min before measurement. Brachial-ankle Pulse Wave Velocity (baPWV), brachial Systolic Blood Pressure (SBP), brachial Diastolic Blood Pressure (DBP) were obtained by using an automated vascular testing device (VP-1000 plus, Omron Healthcare, Kyoto, Japan). By following the guideline [14], blood pressure was determined by the average of three visits to the laboratory pre- and post-exercise training; arterial stiffness measurements in the last visit were made in duplicate, and average values were used for subsequent analyses.

\subsubsection{Arterial hemodynamics}

Longitudinal images of the common carotid or femoral artery $1-2 \mathrm{~cm}$ proximal to the bifurcation were obtained noninvasively in the supine position also by using an ultrasound machine (Sonosite Ultrasound System, Bothell, WA, USA). Vessel diameter was determined at a perpendicular angle along the axis of the scanned area, and blood velocity was assessed with the transducer appropriately positioned to maintain an insonation angle at $60^{\circ}$ or less. Images were analyzed using image analysis software that included at least 10 consecutive waves in the analysis (ImageJ, NIH, Bethesda, MD, USA). Mean blood velocity $\left(V_{\text {mean }}, \mathrm{cm} / \mathrm{s}\right)$ was calculated using the following formula: $\left(\left(1 / 4 V_{\max }\right)+\left(1 / 6 V_{\min }\right)\right) / \mathrm{HR}$ (beats per second) [15]. Blood flow was calculated as $V_{\text {mean }} \times \pi \times$ (femoral diastolic diameter $/ 2)^{2} \times 60, \mathrm{ml} / \mathrm{min}$ [16]. An independent blinded investigator performed all ultrasound imaging and analyses, and day-to-day coefficients of variation for femoral artery diameter and velocity in our laboratory are $1.62 \%$ and $1 \%$, respectively.

Carotid blood pressure, carotid and femoral artery compliance, femoral $\beta$-stiffness index, Ankle-Brachial Index (ABI), carotid and femoral blood flow, as well as leg resistance, were measured noninvasively after subjects rested well in the supine position. Noninvasive pulse tonometer (SPT-301, Millar, Houston, TX, USA) was connected to a physiological signaling processing system (MP36, Biopac, Goleta, CA, USA) to detect pressure waveforms on carotid and femoral artery. Carotid-femoral PWV (cfPWV) was calculated from the traveling distance and foot-to-foot wave transit time between two arterial waveforms [17]. Carotid and femoral blood pressure were measured by calibrating pressure waveforms by brachial blood pressure values, as previously described [17]. The combination of ultrasound imaging of common carotid and femoral artery with simultaneous applanation tonometric-derived arterial waveforms from the contralateral artery permits noninvasive determination of arterial compliance and $\beta$-stiffness index by using the following equations: $\left[\left(D_{1}-D_{0}\right) / D_{0}\right] /\left[2\left(P_{1}-P_{0}\right)\right] \pi D_{0}^{2}$ and $\left(\ln P_{1} / P_{0}\right) /\left[\left(D_{1}-D_{0}\right) / D_{0}\right]$. $D_{1}$ and $D_{0}$ are the maximum and minimum vessel diameters measured from intima to intima, and $P_{1}$ and $P_{0}$ are the highest and lowest blood pressure at carotid and femoral artery after adjusting for hold-down pressure. An independent investigator analyzed an average of 10 heart cycles. Leg resistance was calculated as mean arterial pressure (MAP)/femoral blood flow.

\subsubsection{Muscular fitness}

A 30-s sit-to-stand and a 30-s dominant arm curl test were performed to assess leg muscle power and arm muscle endurance before and after exercise training.

\subsubsection{Statistical analyses}

Statistical analyses were performed using Graph Pad Prism 8.0 (La Jolla, CA, USA). All data are reported as mean \pm SEM. Two-way mixed ANOVA with a Bonferroni post hoc analysis was used to 
determine the effects of exercise and time on measured variables. Significance was set $a$ priori at $p<0.05$.

\section{RESULTS}

Overall, 37 subjects were recruited into the intervention, and three subjects in the control group did not complete the intervention due to personal reasons (i.e., a lack of time). Therefore, a total of 34 subjects completed the study, and selected subject characteristics were presented in Table 1. There were no significant group differences in measured variables pre- and post-training. Compared with the baseline, no significant difference was found in most of the measured variables, except the walking group significantly increased $\mathrm{VO}_{2} \max (p=0.02)$, and the core group significantly improve their arm curl and sit-to-stand performance respectively ( $p=0.03$ and 0.04$)$.

There was no group and time difference in cfPWV (Figure 2A) and ABI (Figure 2C) in all groups. The core group was demonstrated to have higher baPWV compared with the control $(p=0.01)$, and the significance was not detected after training (Figure $2 \mathrm{~B}$ ). Post-training femoral blood flow in the core group significantly increased $(p=0.03)$, and it was higher than that of the control $(p=0.02)$ (Figure 3B). On the other hand, the walking group was demonstrated to significantly lower baPWV $(p=0.04)$ and leg resistance $(p=0.02)$ (Figure $3 \mathrm{~A})$, as well as to increase the femoral artery compliance $(p=0.04)$ after exercise training (Figure $3 \mathrm{C})$. Femoral blood flow also tended to increase after training $(p=0.08)$. Post-exercise training femoral compliance was also significantly higher than that of the control group $(p=0.03)$. For hemodynamic changes, there was no significant difference in heart rate, blood pressure responses, as well as femoral shear rate, diameter, and velocity changes pre- and post-training (Table 2).

\section{DISCUSSION}

The primary findings of this study are as follows: (1) a short-term standing core rotational exercise training on this device could enhance femoral blood flow, arm muscle endurance and leg muscle power; however, it did not contribute to vascular function improvement. (2) Traditional limb-associated walking exercise appears to more effective in improving leg blood flow and vascular functions compared with core exercise in older adults. (3) Eight weeks of endurance exercise training appears not to elicit significant blood pressure reduction and vascular remodeling changes.

Age-related loss of muscle mass or oxygen demand has been demonstrated associated with blood flow reduction in healthy adults; exercise is believed an effective strategy to attenuate such aging deterioration. However, evidence of which exercise training affects basal limb blood flow is not consistent. Cross-sectional studies showed that resistance-trained [3] could better preserve limb blood flow with aging, yet endurance-trained people did not appear to have a higher blood flow level than age-matched counterparts [18]. On the other hand, intervention studies [6,19-21], but not all [22], demonstrated conventional endurance and resistance exercise training could enhance basal limb blood flow in healthy middle-aged to older adults. In the present study, we included a walking exercise group as an active control. Although the magnitude of blood flow increase in the walking group $(\sim 21 \%)$ was somewhat lower than the recently published study ( 31\%) [19], our result was still consistent with findings from previous studies $[19,21,23]$.

In the core exercise group, on the other hand, blood flow was also found to increase after training, and its improvement $(\sim 19 \%)$ was comparable to that of the walking group. Interestingly, despite no between- and within-group difference shown in Table 2, a tendency that both vessel diameter and velocity increased from baseline in

Table 1 Selected subject characteristics

\begin{tabular}{|c|c|c|c|c|c|c|}
\hline & \multicolumn{2}{|c|}{ Control } & \multicolumn{2}{|c|}{ Walking } & \multicolumn{2}{|c|}{ Core } \\
\hline & Pre & Post & Pre & Post & Pre & Post \\
\hline Male/Female, $n$ & $4 / 5$ & - & $6 / 7$ & - & $6 / 6$ & - \\
\hline Age, years & $70 \pm 3$ & - & $66 \pm 2$ & - & $69 \pm 2.2$ & - \\
\hline Height, cm & $162 \pm 3.0$ & - & $161 \pm 2.4$ & - & $164 \pm 1.9$ & - \\
\hline Weight, kg & $67 \pm 5.2$ & $66 \pm 5.0$ & $62 \pm 3.9$ & $61 \pm 3.5$ & $68 \pm 3.3$ & $68 \pm 3.4$ \\
\hline $\mathrm{BMI}, \mathrm{kg} / \mathrm{m}^{2}$ & $25.4 \pm 1.2$ & $25.0 \pm 1.1$ & $23.8 \pm 1.1$ & $23.5 \pm 1.0$ & $26.5 \pm 1.6$ & $25.2 \pm 0.8$ \\
\hline Percent body fat, $\%$ & $29.6 \pm 1.6$ & $28.9 \pm 1.3$ & $29.5 \pm 2.4$ & $28.5 \pm 2.3$ & $31.6 \pm 2.7$ & $32.8 \pm 2.1$ \\
\hline Total cholesterol, mg/dL & $166.5 \pm 12$ & $163.0 \pm 7.4$ & $210.1 \pm 18.7$ & $201.7 \pm 13.4$ & $206.4 \pm 18.5$ & $194.9 \pm 16.0$ \\
\hline HDL cholesterol, mg/dL & $50.2 \pm 4.1$ & $46.3 \pm 3.8$ & $56.9 \pm 4.9$ & $58.4 \pm 6.0$ & $58.8 \pm 4.9$ & $60.8 \pm 3.6$ \\
\hline LDL cholesterol, mg/dL & $97 \pm 11$ & $95.2 \pm 6.8$ & $110.2 \pm 16.5$ & $111.5 \pm 9.8$ & $123.7 \pm 15.5$ & $112.8 \pm 13.8$ \\
\hline $\mathrm{HbA1C}, \%$ & $6.6 \pm 0.5$ & $6.4 \pm 0.3$ & $5.7 \pm 0.2$ & $5.7 \pm 0.2$ & $5.6 \pm 0.2$ & $5.7 \pm 0.2$ \\
\hline Fasting glucose, $\mathrm{mg} / \mathrm{dL}$ & $111.2 \pm 16.5$ & $107.7 \pm 14.3$ & $100.2 \pm 5.0$ & $98.7 \pm 4.2$ & $97.4 \pm 5.9$ & $96.9 \pm 6.2$ \\
\hline Insulin, IU/mL & $7.9 \pm 1.5$ & $8.3 \pm 1.5$ & $11.0 \pm 1.7$ & $9.2 \pm 1.0$ & $8.7 \pm 1.0$ & $7.4 \pm 0.7$ \\
\hline HOMA-IR & $2.1 \pm 0.4$ & $2.3 \pm 0.5$ & $2.5 \pm 0.5$ & $2.1 \pm 0.3$ & $2.0 \pm 0.3$ & $1.7 \pm 0.2$ \\
\hline Physical activity, METs & $2656 \pm 960$ & $3197 \pm 916$ & $2414 \pm 775$ & $3033 \pm 912$ & $2227 \pm 516$ & $3807 \pm 828$ \\
\hline $\mathrm{VO}_{2} \max , \mathrm{ml} / \mathrm{kg} / \mathrm{min}$ & $32 \pm 4$ & $32 \pm 5$ & $34 \pm 1$ & $37 \pm 1^{*}$ & $33 \pm 2$ & $34 \pm 2$ \\
\hline Calorie intake, kcal/day & $1564 \pm 244$ & $1574 \pm 188$ & $1599 \pm 147$ & $1527 \pm 102$ & $1650 \pm 70$ & $1679 \pm 85$ \\
\hline Carbohydrate intake, \% & $54 \pm 3$ & $55 \pm 3$ & $54 \pm 2$ & $52 \pm 2$ & $53 \pm 2$ & $48 \pm 2$ \\
\hline Protein intake, $\%$ & $16 \pm 2$ & $15 \pm 1$ & $16 \pm 1$ & $17 \pm 1$ & $17 \pm 1$ & $16 \pm 1$ \\
\hline Fat intake, $\%$ & $32 \pm 3$ & $30 \pm 2$ & $32 \pm 2$ & $33 \pm 2$ & $31 \pm 2$ & $35 \pm 1$ \\
\hline Arm curl, reps & $14 \pm 2$ & $15 \pm 2$ & $17 \pm 1$ & $17 \pm 3$ & $14 \pm 2$ & $18 \pm 2^{*}$ \\
\hline Sit-to-stand, reps & $16 \pm 2$ & $17 \pm 2$ & $18 \pm 1$ & $19 \pm 1$ & $17 \pm 2$ & $20 \pm 1^{*}$ \\
\hline
\end{tabular}

$p<0.05$. Value $=$ Mean \pm SEM. HDL, high-intensity lipoprotein; LDL, low-intensity lipoprotein. 
है
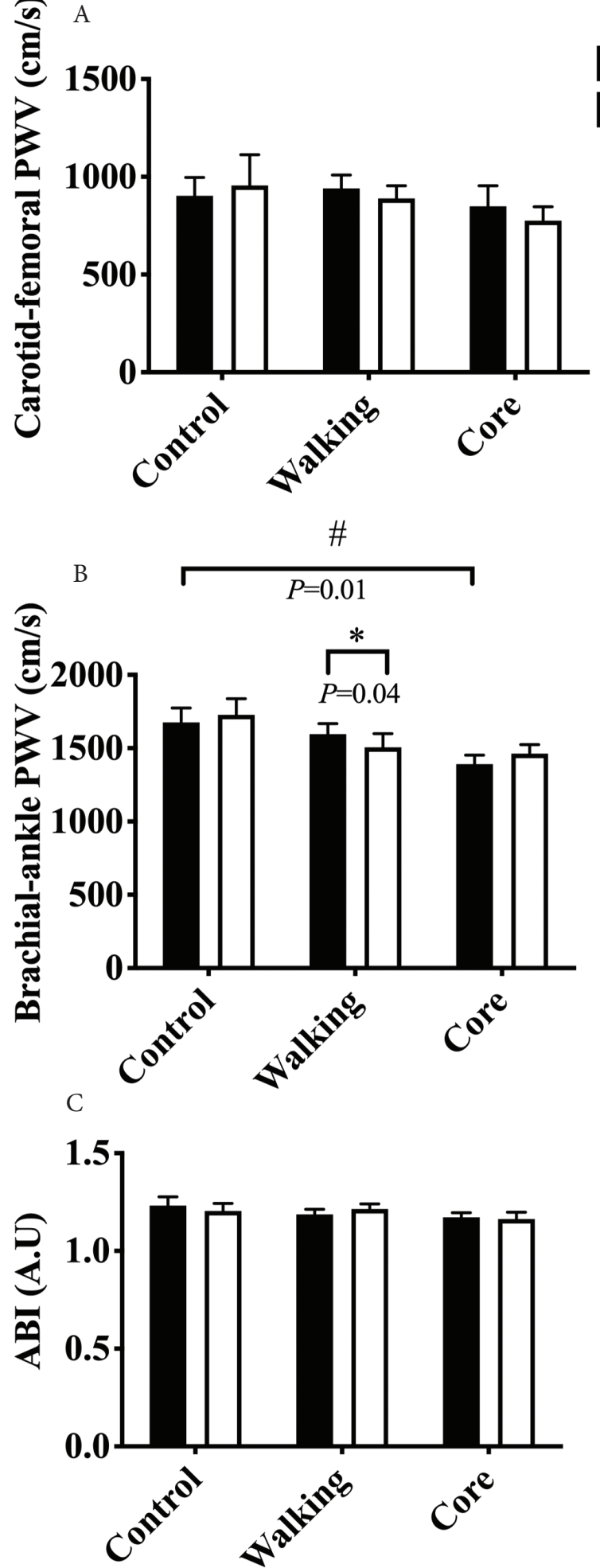

Figure 2 Carotid-femoral (A), brachial-ankle pulse wave velocity (B), and ankle-brachial index $(\mathrm{C})$ changes pre- and post-training.

the walking group was observed, whereas femoral velocity tended to increase in the core exercise group. We speculated it might be due to the difference between exercising muscle location and the size of muscle mass. Indeed, arterial remodeling was found predominantly in exercising muscles that contribute to "ascending vasodilatory" responses in the vasculature [24]. The increase of blood velocity in the core group might reflect the higher resting
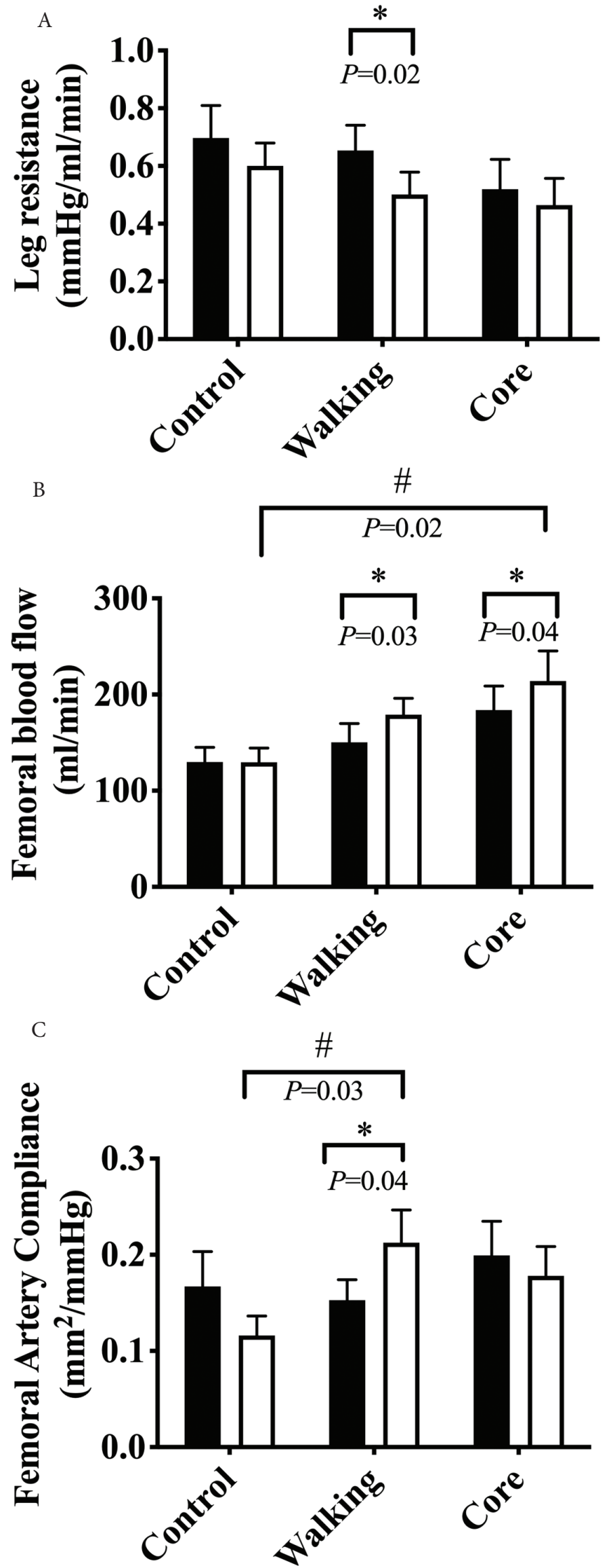

Figure 3 Leg resistance (A), femoral blood flow (B), and femoral artery compliance $(\mathrm{C})$ change pre- and post-training. 
Table 2 Blood pressure and leg hemodynamic changes pre- and post-training

\begin{tabular}{|c|c|c|c|c|c|c|}
\hline & \multicolumn{2}{|c|}{ Control } & \multicolumn{2}{|c|}{ Walking } & \multicolumn{2}{|c|}{ Core } \\
\hline & Pre & Post & Pre & Post & Pre & Post \\
\hline \multicolumn{7}{|l|}{ Blood pressure } \\
\hline Heart rate, bpm & $69 \pm 4$ & $68 \pm 4$ & $69 \pm 2$ & $65 \pm 2$ & $63 \pm 5$ & $64 \pm 5$ \\
\hline Brachial SBP, mmHg & $122 \pm 5$ & $121 \pm 6$ & $126 \pm 3$ & $121 \pm 3$ & $125 \pm 3$ & $125 \pm 5$ \\
\hline Brachial DBP, mmHg & $73 \pm 3$ & $74 \pm 4$ & $75 \pm 3$ & $72 \pm 3$ & $75 \pm 2$ & $73 \pm 3$ \\
\hline Carotid SBP, mmHg & $118 \pm 4$ & $119 \pm 4$ & $116 \pm 3$ & $115 \pm 4$ & $115 \pm 3$ & $116 \pm 4$ \\
\hline Carotid DBP, mmHg & $82 \pm 3$ & $77 \pm 2$ & $79 \pm 2$ & $74 \pm 3$ & $77 \pm 3$ & $80 \pm 3$ \\
\hline Carotid PP, mmHg & $40 \pm 4$ & $40 \pm 4$ & $36 \pm 2$ & $37 \pm 4$ & $37 \pm 2$ & $38 \pm 2$ \\
\hline \multicolumn{7}{|l|}{ Leg hemodynamics } \\
\hline Ankle SBP, mmHg & $155 \pm 9$ & $152 \pm 10$ & $154 \pm 5$ & $155 \pm 6$ & $142 \pm 5$ & $143 \pm 6$ \\
\hline Ankle MAP, mmHg & $106 \pm 6$ & $105 \pm 7$ & $101 \pm 3$ & $98 \pm 4$ & $92 \pm 3$ & $94 \pm 3$ \\
\hline Femoral shear rate, $\mathrm{L} / \mathrm{s}$ & $66.5 \pm 7.7$ & $69.0 \pm 9.6$ & $53.7 \pm 2.8$ & $55.6 \pm 4.3$ & $59.7 \pm 6.7$ & $72.3 \pm 10.4$ \\
\hline Femoral diameter, $\mathrm{cm}$ & $0.90 \pm 0.04$ & $0.88 \pm 0.04$ & $0.96 \pm 0.04$ & $1.01 \pm 0.05$ & $1.02 \pm 0.07$ & $1.02 \pm 0.07$ \\
\hline Femoral velocity, $\mathrm{cm} / \mathrm{s}$ & $14.7 \pm 1.4$ & $14.8 \pm 1.8$ & $13.1 \pm 0.9$ & $14.2 \pm 1.1$ & $14.5 \pm 1.1$ & $17.5 \pm 2.2$ \\
\hline
\end{tabular}

PP, pulse pressure.

metabolic demand as the result of resistance endurance training. Also, exercising with larger muscle mass was found associated longer parasympathetic dominance [25], all of which suggested the extent to which vasodilatory responses induced by walking exercise would be greater than core exercise after training. Therefore, it might not be surprising that the walking group not only significantly increased femoral blood flow, but also femoral artery compliance, and systemic arterial stiffness (baPWV) independent of blood pressure and metabolic biomarker changes. Collectively, results from this study support the findings that walking is a practical choice of exercise to improve overall vascular function for middle-aged to older adults [4,19,26].

Endurance exercise has been demonstrated to effective lower blood pressure by many meta-analysis studies [7,26,27]; however, in the present study, walking did not elicit significant changes on central and peripheral blood pressure, despite a greater reduction on brachial SBP and DBP was observed. Such difference could be due to subjects' baseline blood pressure and lower training stimulus. Indeed, meta-analysis evidence indicated SBP might not be reduced after training if participants are normotensive, whereas DBP were most likely to be affected by endurance training regardless of initial blood pressure level $[7,26]$. In addition, our training intensity might be lower than the recommendation [27], despite weekly training time ( $>150 \mathrm{~min} /$ week or $<150 \mathrm{~min} /$ week) appear not affect the training effects in healthy population $[7,26]$. It should be noted walking exercise intensity was determined by matching with core exercise in the present study; the results from core exercise agreed with the finding that combined exercise training is less effective than endurance exercise alone [7].

The present study does not investigate the mechanisms underlying the increased femoral blood flow in core exercise, and we could only speculate the possible mechanisms. First, the changes in blood flow might result from an elevated whole-body metabolic rate after training. We cannot exclude the possibility that muscle mass might play a role since a low-intensity resistance endurance training has been demonstrated to increase muscle mass, as well as blood flow [20]. However, we did not quantify muscle mass changes, and our intervention period was relatively short to induce muscle hypertrophy. Another possibility is that vasodilator substances from arms and legs, in addition to core muscles, might have also been released into the system; thus, blood flow increased. Indeed, blood flow has been shown to increase even in non-exercising muscles [5], and systemic endothelium-derived vasodilator substance from both active and non-active muscles might contribute to such changes. Repeated increase in flow is believed to act on the endothelium to enhance nitric oxide synthase expression [5] and reduce vasoconstrictor tone [21] after exercise training.

Core-biased exercise in the present study is a whole-body resistance endurance exercise consisting of a 30-min trunk rotational movement under low resistance tension, which consists of endurance and strength training components. Electromyographic analysis performed by a previous study using similar exercise determined rectus abdominis, obliquus externus abdominis, longissimus, and multifidus muscles may have been recruited [8]. The notion of combining endurance and strength components into training is designed to seek further health benefits, compared with single training mode alone [28]. Despite our intervention only last 8 weeks, we demonstrated this type of exercise also benefits leg muscle power and arm muscular endurance, as the result of neuromuscular adaptation, which could be applied for clinical use as an exercise modality for older populations.

Some limitations warrant caution when interpreting the results of our study. We only included middle-aged to older healthy sedentary participants in the study, which limits our findings generalized to other populations, such as stroke patients who can also benefit from core exercise training [29]. Future research is, therefore, needed to investigate its efficacy in the disease population. Furthermore, as discussed, the increase in blood flow after training can be attributed to many physiological adaptations. We did not analyze biomarkers such as nitric oxide, endothelin-1, or catecholamine, which could have provided more mechanistic insights into the differing training effects of exercise mode on blood flow and vascular function. However, we could exclude the potential influence from the metabolic and physical activity factors as they were well controlled pre- and post-training in the present study.

In summary, to our knowledge, this study is the first to demonstrate core-biased exercise training could enhance not only femoral blood flow but also limb muscular fitness in healthy middle-aged to older adults. Walking exercise, on the other hand, could exert a more pronounced improvement in vascular function after short-term exercise training and all of which could have significant implications to the public in exercise participation. 


\section{CONFLICTS OF INTEREST}

The authors declare they have no conflicts of interest.

\section{AUTHORS' CONTRIBUTION}

HL and SW study conceptualization, data collection, and writing (original draft) the manuscript. HL, HC and JS formal analysis, writing, review and editing the manuscript.

\section{ACKNOWLEDGMENTS}

This study was reviewed and approved by the Institutional Review Board of National Taiwan University Hospital (201605015RIND). We would like to thank all the participants and image analysis assistance of Shiou-Shu, Huang, and Yun-Hsuan, Chen. This study was supported by a research grant from the Ministry of Science and Technology in Taiwan (MOST 103-2410-H-002-196-MY2).

\section{REFERENCES}

[1] Dinenno FA, Jones PP, Seals DR, Tanaka H. Limb blood flow and vascular conductance are reduced with age in healthy humans: relation to elevations in sympathetic nerve activity and declines in oxygen demand. Circulation 1999;100:164-70.

[2] Restaino RM, Walsh LK, Morishima T, Vranish JR, MartinezLemus LA, Fadel PJ, et al. Endothelial dysfunction following prolonged sitting is mediated by a reduction in shear stress. Am J Physiol Heart Circ Physiol 2016;310:H648-H53.

[3] Miyachi M, Tanaka H, Kawano H, Okajima M, Tabata I. Lack of age-related decreases in basal whole leg blood flow in resistancetrained men. J Appl Physiol 2005;99:1384-90.

[4] Seals DR, Desouza CA, Donato AJ, Tanaka H. Habitual exercise and arterial aging. J Appl Physiol (1985) 2008;105:1323-32.

[5] Tanaka H, Shimizu S, Ohmori F, Muraoka Y, Kumagai M, Yoshizawa $\mathrm{M}$, et al. Increases in blood flow and shear stress to nonworking limbs during incremental exercise. Med Sci Sports Exerc 2006;38:81-5.

[6] Anton MM, Cortez-Cooper MY, DeVan AE, Neidre DB, Cook JN, Tanaka H. Resistance training increases basal limb blood flow and vascular conductance in aging humans. J Appl Physiol (1985) 2006;101:1351-5.

[7] Cornelissen VA, Smart NA. Exercise training for blood pressure: a systematic review and meta-analysis. J Am Heart Assoc 2013;2:e004473.

[8] Tarnanen SP, Siekkinen KM, Häkkinen AH, Mälkiä EA, Kautiainen HJ, Ylinen JJ. Core muscle activation during dynamic upper limb exercises in women. J Strength Cond Res 2012;26:3217-24.

[9] Granacher U, Gollhofer A, Hortobágyi T, Kressig RW, Muehlbauer T. The importance of trunk muscle strength for balance, functional performance, and fall prevention in seniors: a systematic review. Sports Med 2013;43:627-41.

[10] Akuthota V, Nadler SF. Core strengthening. Arch Phys Med Rehabil 2004;85:S86-S92.

[11] Ohkoshi Y, Yasuda K, Kaneda K, Wada T, Yamanaka M. Biomechanical analysis of rehabilitation in the standing position. Am J Sports Med 1991;19:605-11.

[12] Vaitkevicius PV, Fleg JL, Engel JH, O'Connor FC, Wright JG, Lakatta LE, et al. Effects of age and aerobic capacity on arterial stiffness in healthy adults. Circulation 1993;88: 1456-62.

[13] American College of Sports Medicine. ACSM's Guidelines for Exercise Testing and Prescription. Baltimore, MD: Lippincott Williams \& Wilkins; 2006.

[14] Muntner P, Shimbo D, Carey RM, Charleston JB, Gaillard T, Misra S, et al. Measurement of blood pressure in humans: a scientific statement from the American Heart Association. Hypertension 2019;73:e35-e66.

[15] Groothuis JT, Boot CRL, Houtman S, van Langen H, Hopman MTE. Does peripheral nerve degeneration affect circulatory responses to head-up tilt in spinal cord-injured individuals? Clin Auton Res 2005;15:99-106.

[16] Donato AJ, Uberoi A, Walter Wray D, Nishiyama S, Lawrenson L, Richardson RS. Differential effects of aging on limb blood flow in humans. Am J Physiol Heart Circ Physiol 2006;290:H272-H8.

[17] Tanaka H, DeSouza CA, Seals DR. Absence of age-related increase in central arterial stiffness in physically active women. Arterioscler Thromb Vasc Biol 1998;18:127-32.

[18] Dinenno FA, Seals DR, DeSouza CA, Tanaka H. Age-related decreases in basal limb blood flow in humans: time course, determinants and habitual exercise effects. J Physiol 2001;531:573-9.

[19] Hurley DM, Williams ER, Cross JM, Riedinger BR, Meyer RA, Abela GS, et al. Aerobic exercise improves microvascular function in older adults. Med Sci Sports Exerc 2019;51:773-81.

[20] Tanimoto M, Kawano H, Gando Y, Sanada K, Yamamoto K, Ishii N, et al. Low-intensity resistance training with slow movement and tonic force generation increases basal limb blood flow. Clin Physiol Funct Imaging 2009;29:128-35.

[21] Thijssen DHJ, Rongen GA, van Dijk A, Smits P, Hopman MTE. Enhanced endothelin-1-mediated leg vascular tone in healthy older subjects. J Appl Physiol (1985) 2007;103:852-7.

[22] Phillips B, Williams J, Atherton P, Smith K, Hildebrandt W, Rankin D, et al. Resistance exercise training improves agerelated declines in leg vascular conductance and rejuvenates acute leg blood flow responses to feeding and exercise. J Appl Physiol (1985) 2012;112:347-53.

[23] Carter SE, Draijer R, Holder SM, Brown L, Thijssen DHJ, Hopkins ND. Effect of different walking break strategies on superficial femoral artery endothelial function. Physiol Rep 2019;7:e14190.

[24] Wray DW, Uberoi A, Lawrenson L, Richardson RS. Heterogeneous limb vascular responsiveness to shear stimuli during dynamic exercise in humans. J Appl Physiol (1985) 2005;99:81-6.

[25] Matos-Santos L, Farinatti P, Borges PJ, Massaferri R, Monteiro W. Cardiovascular responses to resistance exercise performed with large and small muscle mass. Int J Sports Med 2017; 38:883-9

[26] Murphy MH, Nevill AM, Murtagh EM, Holder RL. The effect of walking on fitness, fatness and resting blood pressure: a metaanalysis of randomised, controlled trials. Prev Med 2007;44:377-85.

[27] Lee LL, Watson MC, Mulvaney CA, Tsai CC, Lo SF. The effect of walking intervention on blood pressure control: a systematic review. Int J Nurs Stud 2010;47:1545-61.

[28] Karavirta L, Häkkinen K, Kauhanen A, Arija-Blázquez A, Sillanpää E, Rinkinen N, et al. Individual responses to combined endurance and strength training in older adults. Med Sci Sports Exerc 2011;43:484-90.

[29] Haruyama K, Kawakami M, Otsuka T. Effect of core stability training on trunk function, standing balance, and mobility in stroke patients: a randomized controlled trial. Neurorehabil Neural Repair 2017;31:240-9. 